\title{
Star Polymers for Gene Delivery
}

Theoni K. Georgiou

\author{
Surfactant \& Colloid Group \\ Department of Chemistry \\ University of Hull \\ Hull, HU6 7RX \\ UNITED KINGDOM
}

Submitted for publication to the

Polymer International

July 2013

e-mail address of author:

T. K. Georgiou:

t.georgiou@hull.ac.uk 


\begin{abstract}
Star polymers are hyperbranched polymers with fascinating properties and self assembly behaviour that have recently attracted a lot of interest in the field of gene delivery. This perspective aims to summarize the latest studies on star polymers as gene delivery vectors. Specifically, to identify and discuss the main synthetic methodologies that were used to fabricate the star polymers and which structural characteristics affect their ability to be used as gene delivery agents.
\end{abstract}

Keywords: polymers; star polymers; in-out star, arm-first star, core-first star, hyperbranched polymers, nanomedicine; gene therapy; gene delivery; DNA delivery; siRNA delivery; molecular weight effect; architecture effect. 


\section{Introduction}

Gene therapy can be used to treat a number of genetic disorders like blindness, diabetes, cystic fibrosis, Parkinson's disease, cancer, and haemophilia. ${ }^{1,2}$ In order for therapy to be achieved, the appropriate therapeutic gene (DNA or siRNA) has to be delivered into the cells. DNA however, is a negatively charged, hydrophilic molecule; thus its delivery into the nucleus of the cell which requires it to pass through the also negatively charged and hydrophobic cell membrane is not feasible. Moreover DNA is enzymatically degradable and needs to be protected until it reaches the nucleus of the cells. Consequently gene delivery vesicles (also called vectors or carriers or agents) have been developed. ${ }^{1-7}$ The first vectors trialed in gene delivery were viruses, which are nature's way of carrying genes. ${ }^{6,7}$ Viruses though cause immune response and thus non-viral viruses started to be developed. ${ }^{6-9}$ Many of these non-viral vectors are polymer-based, because polymers are easy to tailor, cost-effective and safer. $6,7,9,10$ This explains why over the last three decades more than three thousand studies have been published on polymers for gene delivery.

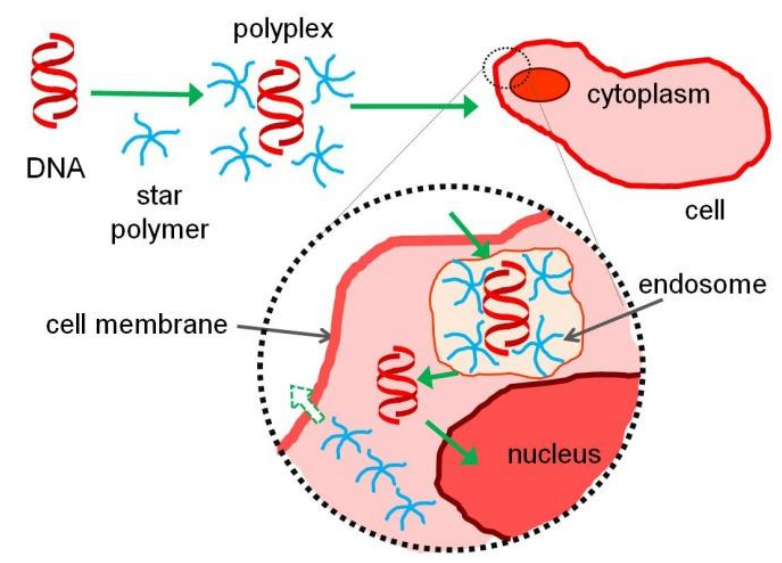

Figure 1: DNA delivery using a cationic star polymer.

Most of the polymers used for gene delivery are cationic. The delivery of the genetic material (a procedure also called transfection) happens in five steps when using a cationic polymer vector (illustrated in Figure 1): 
i. DNA / polymer complexation (also called DNA condensation). The cationic polymer electrostatically binds the negatively charged DNA.

ii. The DNA / polymer complex (also called polyplex) passes through the cell membrane into the cell, usually by endocytosis.

iii. Endosomal release of the polyplex or lysosomal degradation in the cytoplasm. The polyplex enters the cytoplasm usually in an endosome (depending on the cell type and the type of entry). The polyplex is then released from the endosome which has a $\mathrm{pH} \sim$ 6 before the endosome matures to a lysosome $\mathrm{pH} \sim 5$ to avoid enzymatic degradation.

iv. Cytosolic transport to the nucleus. The polyplex or DNA (if it has already been released from the complex) travels through the cytoplasm to reach the nucleus.

v. Transfer into the nucleus. This can happen by two paths: 1) during mitosis when the nuclear membrane dissembles and large molecules can enter the nucleus and 2) through nuclear pore complexes. With the later, larger molecules they require specific targeting so they can be recognised by the receptors on the nuclear membrane.

Thus, an ideal cationic polymer vector should be water-soluble, non-toxic and be able to bind the DNA effectively, but not too strongly, so it will be able to release the DNA in the cytoplasm. It will also be desirable to be biodegradable and have targeting moieties (groups) that will ensure delivery to the desired area. The chemical structures of common cationic polymers that have been used in gene delivery are shown in Figure 2. These include polyethylenimine (PEI), ${ }^{7,8,11-15}$ poly[(2-dimethylamino)ethyl methacrylate] (pDMAEMA), ${ }^{11-}$ 21 poly(L-lysine) (PLL) $)^{7,} 22$ and poly(N,N-dimethylaminopropyl acrylamide) (pDMAPAAm) $)^{23-27}$. The chemical structure of poly(ethylene glycol) (PEG) is also shown in Figure 2 because it is a common chemical group to attach to polymeric carriers to increase their solubility in water and their biocompatibility. 


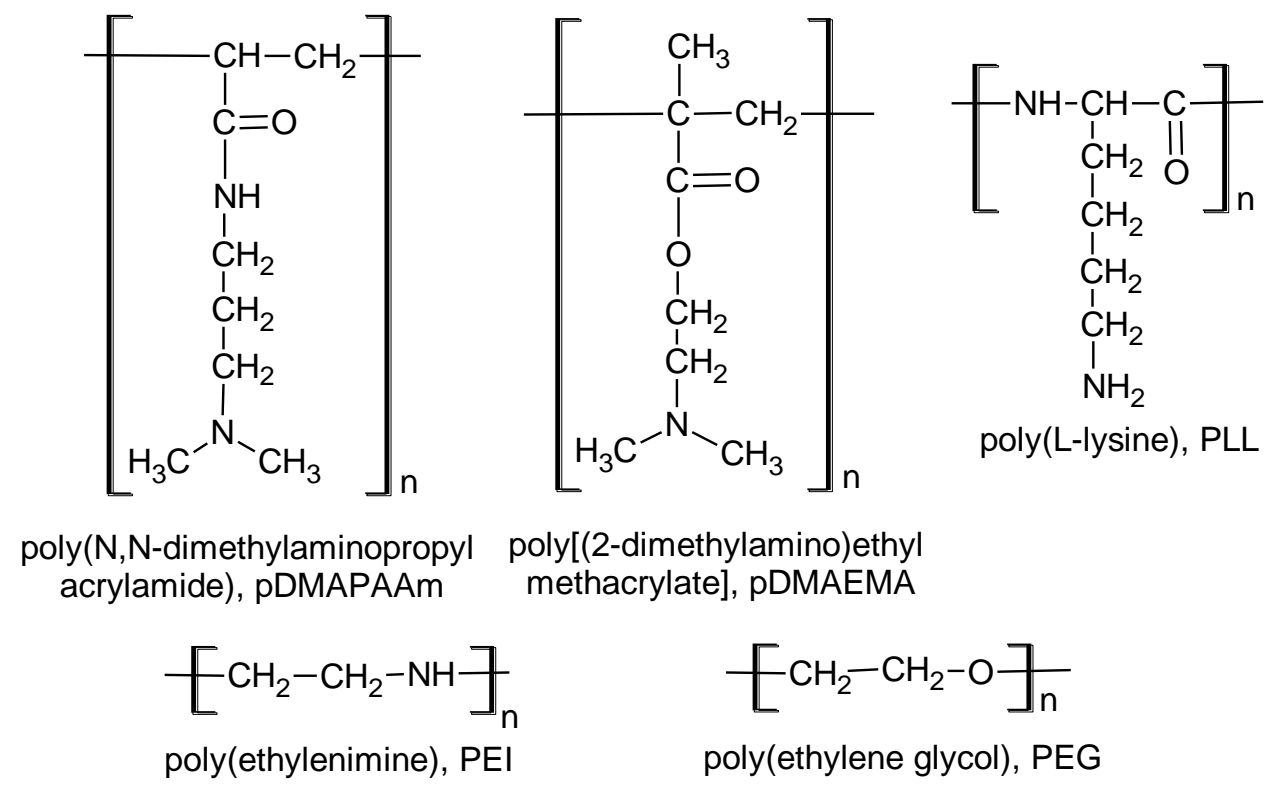

Figure 2: Chemical structures of common components of star-like polymeric DNA carriers.

Numerous types of polymers have been trialed in gene delivery; polymers with different structural characteristics; different chemistry, composition, topology and architecture. ${ }^{27-29}$ This perspective focuses on a specific type of polymer vectors that have a star-like architecture, which has shown promising activity. Specifically, star polymers have exhibited better transfection efficiency when compared to their linear or branched counterparts. ${ }^{15,23,30-32}$ This has been attributed to superior DNA condensation ability of the star polymers in forming spherical polyplexes ${ }^{32}$ that are assumed to enter the cell more easily. Interestingly it was confirmed by theoretical predictions that star polymer/DNA complexes have a spherical shape and that the DNA is positioned more in the core of the complex. ${ }^{33}$ This means that the DNA will be protected more efficiently from enzymatic degradation until its release.

\section{Synthetic Methodologies}

There are three methodologies that have been used to synthesized star polymers for gene delivery which are schematically illustrated in Fig. 3. The first method is the "arm-first" approach where the linear branches of the polymer are synthesised first using a "living" or a 
"controlled" polymerization technique and then a bifunctional cross-linker is used to interconnect the "arms" to produce star polymers. ${ }^{11-15,30,34}$ One disadvantage of this method is that the synthesized star polymers do not have a known number of arms, a defined functionality, and that is why the polydispersity (PDI) can vary from 1.1 to 1.5 , depending on the polymerization method and the monomer used. Furthermore, linear polymers that do not interconnect to produce the star polymer remain in solution so further purification of the star polymer is sometimes needed. Another disadvantage is that when the degree of polymerization of the arms is low it is possible for the star polymers to interconnect with each other and thus produce crosslinked star polymers with even broader PDIs (around 2). ${ }^{14,15}$ The advantage of this method though is that the position of the monomers can be easily varied when synthesizing star copolymers. Therefore polymers of different architectures like block, statistical and heteroarms star copolymers can easily be synthesized in a one-pot procedure with sequential living polymerization. ${ }^{11,13}$ Also, if a degradable crosslinker is used then star polymers with a degradable core are produced which have a significant advantage since degradability has been shown to reduce the toxicity of cationic polymer vectors.

The second methodology to synthesize star polymers is to use a multifunctional initiator from which the "arms" (branches) grow from using a "living" or a "controlled" polymerization technique. $^{23-26,31,32,35-46}$ This approach produces star polymers with a known functionality (number of arms) equal to the functionality of the initiator and narrower PDIs than the first synthetic methodology. The challenge in this approach is the use of the right initiator that will bear initiating sites (functional groups) with the same reactivity so all the arms will "grow" with the same rate and have the same degree of polymerization. Often, the multifunctional initiators have to be in-house synthesized.

The third approach is to synthesize star polymers by using a series of conjugation/coupling reactions. ${ }^{41,45,47-51}$ The functionality of the synthesized star polymer will be equal to that of 
the multifunctional coupling agent/precursor that will be used. The disadvantage of this method is that the attachment of the linear polymers to the multifunctional compound, that will act as the core of the arm, can be time consuming or/and will not go to full conversion. ${ }^{52}$ However, with the recent development of "click" chemistry this is no longer as a major problem as it used to be. It can still be a time consuming, multi-pot procedure though, especially to synthesize block copolymers, unlike the first two approaches that can potentially be done as a one-pot procedure if a "living" polymerisation method is used.

(a)

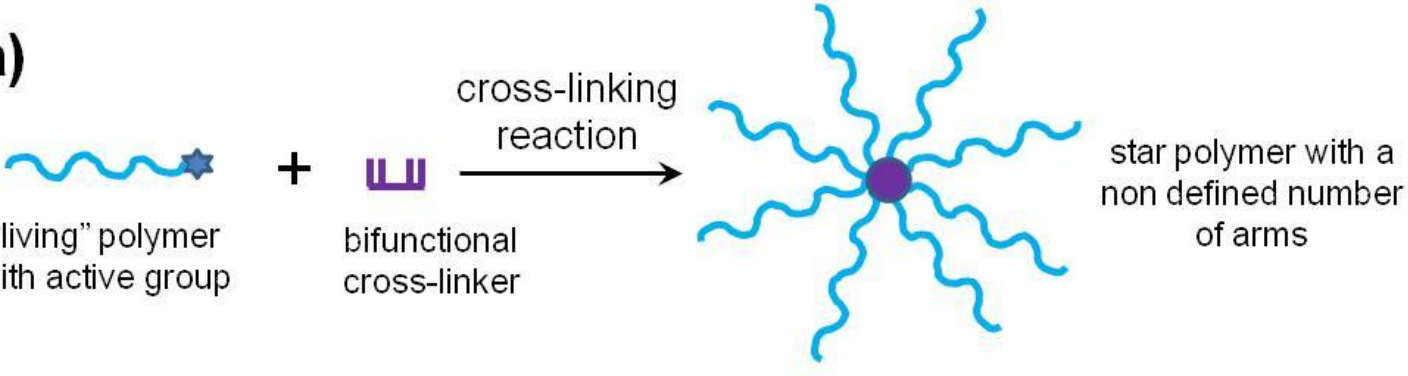

(b)

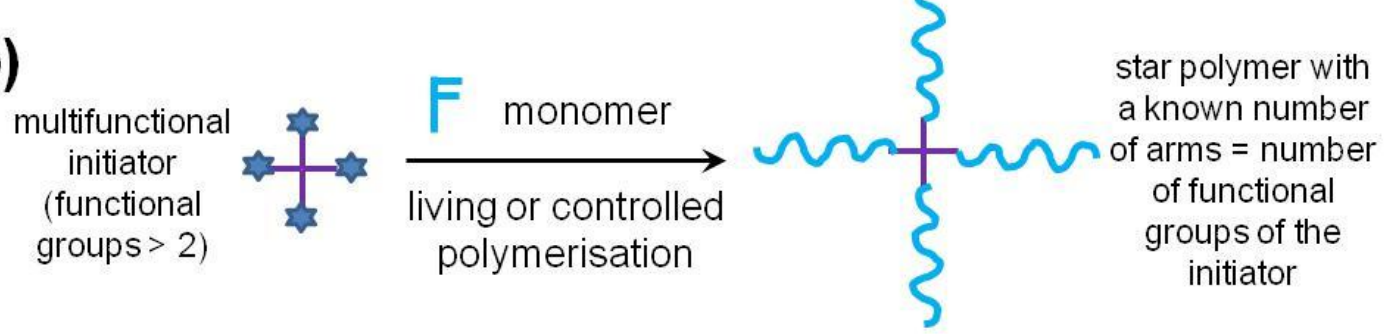

(c) polymer with functional group

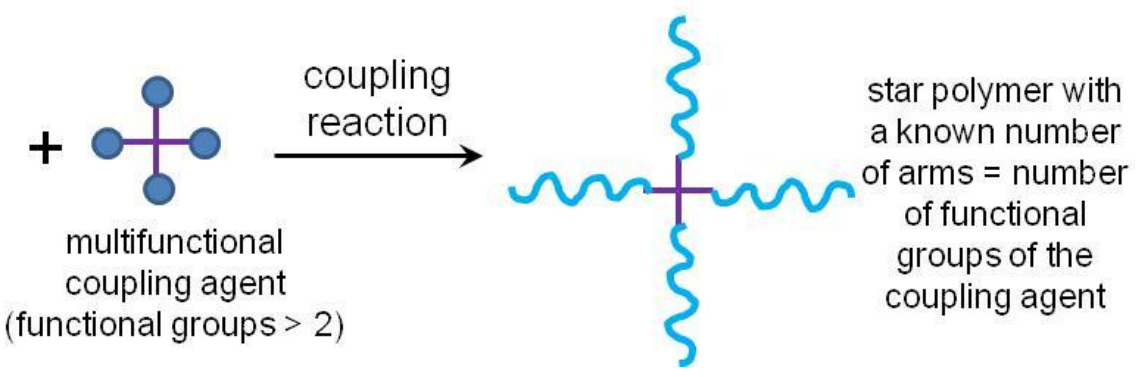

Figure 3: The three main different methods used to synthesize star polymers for gene delivery: (a) the "arm-first" methodology (b) "core-first" methodology and (c) by conjugation/coupling reactions. 
Thus, depending on which methodology is chosen star polymers of different functionalities and architectures can be synthesized. These structural characteristics of the star polymers can, as it will be discussed below, significantly affect their ability to deliver the genetic material.

\section{Structure/Activity Relationship}

The first study of star polymers in gene delivery was performed by Georgiou et al. and in this study the effect of the molecular weight (MW) on the transfection efficiency and cell viability was investigated. ${ }^{12}$ The cell viability reduced as the size of the star polymer increased and this was also observed in later studies. ${ }^{24,26,36,43,46,49}$ This is attributed to two reasons: 1 ) larger size star polymers produce larger polyplexes that are more toxic because they have a stronger tendency to bind negatively charged peptides and eventually precipitate with the cytosol or/and 2) larger star polymers can destabilise the cell membrane, that causes cell death, more effectively than smaller cationic polymers.

However, when trying to compare studies on how the MW (size) of the star polymers affects the transfection efficiency caution should be taken, not just because no direct comparison of studies using different cell lines and transfection protocols should be made, but also because the MW of star polymers can be varied by changing two parameters: the length of the arms or/and the number of branches. Thus it is not straightforward to conclude how the MW affects the transfection efficiency. For example when comparing two initial studies on how the MW affects the gene delivery ability of the star polymers conflicting results are reported. The study by Georgiou et $\mathrm{al}^{12}$ where DMAEMA based star polymers from $44 \mathrm{k}$ to $177 \mathrm{k} \mathrm{g}$ $\mathrm{mol}^{-1}$ were investigated reported that the transfection efficiency decreased with increasing the MW and the study performed by Nakayama's group ${ }^{26}$ where DMAPAAm based star polymers with MWs from $23 \mathrm{k}$ to $73 \mathrm{k} \mathrm{g} \mathrm{mol}^{-1}$ reports that the gene delivery increased with increasing the MW. However, when carefully considering that cell viability is significantly decreased when increasing the MW and that will have a major role in the overall transfection 
efficieny of the polymer, one can conclude that there is an optimum MW for star polymers for gene delivery. This optimum MW is probably an intermediate MW around 50 to $100 \mathrm{~kg}$ mol $^{-1}$ depending on the chemical structure of the cationic star polymer. This was later investigated by Muller's group and their findings supported this argument. ${ }^{43}$

The introduction of a second monomer, a co-monomer, in the polymer structure usually results in increasing the cell viability if the second monomer is hydrophilic and non-ionic. The most common co-monomer is PEG (or a PEG-based monomer) that is known to be biocompatible, non-immunogenic, non-antigenic and it is also FDA approved. Furthermore the PEG component can improve the lifetime of the carrier in vivo through the shielding effect. Specifically the incorporation of a non-ionic hydrophilic segment on the polymer increases the colloidal stability of the polyplexes, reduces the polyplexes interactions with proteins and blood cells, and prolongs the circulation time in the blood stream. A number of

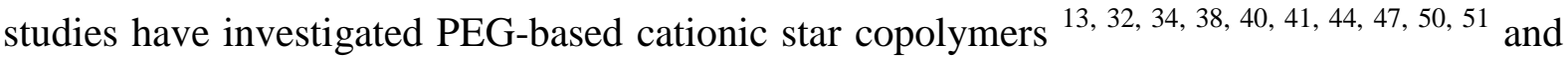
several have confirmed that the incorporation of the PEG component reduces the toxicity when the copolymers were compared with the cationic star homopolymers. ${ }^{13,40,44}$ When hydrophilic anionic groups, specifically methacrylic acid ${ }^{11}$ and folic acid ${ }^{48}$ groups were incorporated into the star polymer structure, increased cell viability or no effect on cell viability was observed, respectively.

Interestingly it is not only the incorporation of the second monomer that affects the vector's transfection ability but also the co-monomer's position. Specifically when star polymers of different architectures but similar composition (monomer ratio) and MW were synthesized the transfection efficiency was significantly affected by the position of the monomers within the polymer structure. ${ }^{11,13}$ It appears that the star copolymers have increased gene delivery activity when the cationic groups are on the outer part of the star. This may be due to the 
better complexation ability of these stars. However further studies are needed to systematically investigate the effect of the architecture and confirm this observation.

Futhermore, in order to improve further the transfection efficiency and reduce the toxicity of star polymer vectors, targeting ${ }^{25,41,48}$ and degradable groups, ${ }^{30,44,48}$ respectively, can been incorporated into the star structure. Targeting groups will assist the polyplex to be delivered to a specific cell type while degradable groups will reduce the toxicity of the polymer because it will be cleaved to smaller polymers in vitro and in vivo and smaller MW polymers have reduced toxicity.

Finally, it is also desirable to introduce imaging agents on the polymeric carriers so the polyplex can be monitored in vivo and their intracellular pathway can be identified. Thus, simultaneous imaging and therapy, theranostics, could be achieved. ${ }^{53}$

\section{Conclusions and Perspectives}

Star polymers have proven to be more promising transfection agents than linear or branched polymers. Due to their compact structure and moderate flexibility they can condensate the DNA and deliver it efficiently into cells. Furthermore the star architecture offers more endfunctional groups where targeting groups can be attached.

Over the last decade, since the first star polymers were trialled as gene delivery vectors, it has become apparent that the structural characteristics of the polymer have a significant effect on their activity. Specifically, structural characteristics like the numbers of arms, as well as the length of the arms, the composition, and the topology of the star polymers affect the toxicity, the DNA complexation ability and the overall transfection efficiency of the polymers. However, further studies are needed in order to establish and confirm how these structural characteristics shape the activity and optimise the carriers. In order for the future studies to be precise and reliable, they should be systematic. This can only be achieved if the produced polymers have well-defined structures and narrow molecular weight distributions. 
Consequently, the use of "living" or "controlled" polymerization techniques will become essential.

\section{References}

1. $\quad$ C. L. Grigsby and K. W. Leong, Journal of the Royal Society Interface, 2010, 7, S67S82.

2. $\quad$ C. K. Payne, Nanomedicine, 2007, 2, 847-860.

3. H. Mok and T. G. Park, Macromol. Biosci., 2012, 12, 40-48.

4. D. Schaffert and E. Wagner, Gene Ther., 2008, 15, 1131-1138.

5. P. Guo, O. Coban, N. M. Snead, J. Trebley, S. Hoeprich, S. Guo and Y. Shu, Adv. Drug Delivery Rev., 2010, 62, 650-666.

6. P. L. Felgner, Sci. Am., 1997, 276, 102-106.

7. T. Merdan, J. Kopeček and T. Kissel, Adv. Drug Delivery Rev., 2002, 54, 715-758.

8. W. T. Godbey and A. G. Mikos, J. Controlled Release, 2001, 72, 115-125.

9. S. O. Han, R. I. Mahato, Y. K. Sung and S. W. Kim, Mol. Ther., 2000, 2, 302-317.

10. A. V. Kabanov, Pharmaceutical Science and Technology Today, 1999, 2, 365-372.

11. T. K. Georgiou, L. A. Phylactou and C. S. Patrickios, Biomacromolecules, 2006, 7, 3505-3512.

12. T. K. Georgiou, M. Vamvakaki, C. S. Patrickios, E. N. Yamasaki and L. A. Phylactou, Biomacromolecules, 2004, 5, 2221-2229.

13. T. K. Georgiou, M. Vamvakaki, L. A. Phylactou and C. S. Patrickios, Biomacromolecules, 2005, 6, 2990-2997.

14. K. S. Pafiti, C. S. Patrickios, T. K. Georgiou, E. N. Yamasaki, N. P. Mastroyiannopoulos and L. A. Phylactou, Eur. Polym. J., 2012, 48, 1422-1430.

15. K. S. Pafiti, N. P. Mastroyiannopoulos, L. A. Phylactou and C. S. Patrickios, Biomacromolecules, 2011, 12, 1468-1479.

16. W. E. Hennink, J. Y. Cherng, W. Jiskoot, H. Talsma and D. J. A. Crommelin, S.T.P. Pharma Sciences, 2001, 11, 11-19.

17. P. Van De Wetering, J. Y. Cherng, H. Talsma, D. J. A. Crommelin and W. E. Hennink, J. Controlled Release, 1998, 53, 145-153.

18. P. Van De Wetering, E. E. Moret, N. M. E. Schuurmans-Nieuwenbroek, M. J. Van Steenbergen and W. E. Hennink, Bioconjugate Chem., 1999, 10, 589-597.

19. P. Van De Wetering, N. M. E. Schuurmans-Nieuwenbroek, W. E. Hennink and G. Storm, J. Gene Med., 1999, 1, 156-165.

20. P. Van De Wetering, N. M. E. Schuurmans-Nieuwenbroek, M. J. Van Steenbergen, D. J. A. Crommelin and W. E. Hennink, J. Controlled Release, 2000, 64, 193-203.

21. N. J. Zuidam, G. Posthuma, E. T. J. De Vries, D. J. A. Crommelin, W. E. Hennink and G. Storm, J. Drug Targeting, 2000, 8, 51-66.

22. S. C. De Smedt, J. Demeester and W. E. Hennink, Pharm. Res., 2000, 17, 113-126.

23. Y. Nakayama, T. Masuda, M. Nagaishi, M. Hayashi, M. Ohira and M. Harada-Shiba, Curr. Drug Delivery, 2005, 2, 53-57.

24. Y. Nakayama, C. Kakei, A. Ishikawa, Y. M. Zhou, Y. Nemoto and K. Uchida, Bioconjugate Chem., 2007, 18, 2037-2044.

25. A. Ishikawa, Y. M. Zhou, N. Kambe and Y. Nakayama, Bioconjugate Chem., 2008, 19, 558-561. 
26. Y. Nemoto, A. Borovkov, Y. M. Zhou, Y. Takewa, E. Tatsumi and Y. Nakayama, Bioconjugate Chem., 2009, 20, 2293-2299.

27. Y. Nakayama, Acc. Chem. Res., 2012, 45, 994-1004.

28. F. J. Xu and W. T. Yang, Prog. Polym. Sci., 2011, 36, 1099-1131.

29. M. Hamidi, M. A. Shahbazi and K. Rostamizadeh, Macromol. Biosci., 2012, 12, 144164.

30. F. Dai, P. Sun, Y. Liu and W. Liu, Biomaterials, 2010, 31, 559-569.

31. A. Schallon, V. Jérôme, A. Walther, C. V. Synatschke, A. H. E. Müller and R. Freitag, Reactive and Functional Polymers, 2010, 70, 1-10.

32. L. Yin, Z. Song, K. H. Kim, N. Zheng, H. Tang, H. Lu, N. Gabrielson and J. Cheng, Biomaterials, 2013, 34, 2340-2349.

33. J. Ziebarth and Y. Wang, J. Phys. Chem. B, 2010, 114, 6225-6232.

34. H. Y. Cho, A. Srinivasan, J. Hong, E. Hsu, S. Liu, A. Shrivats, D. Kwak, A. K. Bohaty, H. J. Paik, J. O. Hollinger and K. Matyjaszewski, Biomacromolecules, 2011, 12, 3478-3486.

35. Y. M. Zhou, A. Ishikawa, R. Okahashi, K. Uchida, Y. Nemoto, M. Nakayama and Y. Nakayama, J. Controlled Release, 2007, 123, 239-246.

36. Y. Nemoto, Y. M. Zhou, E. Tatsumi and Y. Nakayama, Bioconjugate Chem., 2008, 19, 2513-2519.

37. M. Yin, K. Ding, R. A. Gropeanu, J. Shen, R. Berger, T. Weil and K. Müllen, Biomacromolecules, 2008, 9, 3231-3238.

38. E. He, C. Y. Yue, F. Simeon, L. H. Zhou, H. P. Too and K. C. Tam, Journal of Biomedical Materials Research - Part A, 2009, 91, 708-718.

39. T. Mori, A. Ishikawa, Y. Nemoto, N. Kambe, M. Sakamoto and Y. Nakayama, Bioconjugate Chem., 2009, 20, 1262-1269.

40. F. J. Xu, Z. X. Zhang, Y. Ping, J. Li, E. T. Kang and K. G. Neon, Biomacromolecules, 2009, 10, 285-293.

41. D. Li, Y. Ping, F. Xu, H. Yu, H. Pan, H. Huang, Q. Wang, G. Tang and J. Li, Biomacromolecules, 2010, 11, 2221-2229.

42. J. Li, Z. Guo, J. Xin, G. Zhao and H. Xiao, Carbohydr. Polym., 2010, 79, 277-283.

43. C. V. Synatschke, A. Schallon, V. Jérôme, R. Freitag and A. H. E. Müller, Biomacromolecules, 2011, 12, 4247-4255.

44. Y. Hu, W. Yuan, N. N. Zhao, J. Ma, W. T. Yang and F. J. Xu, Biomaterials, 2013, 34, 5411-5422.

45. B. Liang, J. J. Deng, F. Yuan, N. Yang, W. Li, J. R. Yin, S. X. Pu, L. C. Xie, C. Gao and L. M. Zhang, Carbohydr. Polym., 2013, 94, 185-192.

46. K. M. Xiu, J. J. Yang, N. N. Zhao, J. S. Li and F. J. Xu, Acta Biomaterialia, 2013, 9, 4726-4733.

47. X. Cai, C. Dong, H. Dong, G. Wang, G. M. Pauletti, X. Pan, H. Wen, I. Mehl, Y. Li and D. Shi, Biomacromolecules, 2012, 13, 1024-1034.

48. F. Zhao, H. Yin, Z. Zhang and J. Li, Biomacromolecules, 2013, 14, 476-484.

49. C. Yang, H. Li, S. H. Goh and J. Li, Biomaterials, 2007, 28, 3245-3254.

50. K. M. Fichter, L. Zhang, K. L. Kiick and T. M. Reineke, Bioconjugate Chem., 2008, 19, 76-88.

51. R. Namgung, J. Kim, K. Singha, C. H. Kim and W. J. Kim, Mol. Pharm., 2009, 6, 1826-1835.

52. N. Hadjichristidis, M. Pitsikalis, S. Pispas and H. Iatrou, Chem. Rev., 2001, 101, 3747-3792.

53. T. Krasia-Christoforou and T. K. Georgiou, J. Mater. Chem. B, 2013, 1, 3002-3025. 